\title{
Residue class fields of lattice-ordered algebras *
}

by

M. Henriksen, J. R. Isbell and D. G. Johnson (Lafayette, Ind.)

This paper is a continuation of [5], and is concerned with the structure of the residue class fields of the $\Phi$-algebras introduced and studied in that paper. These are archimedean lattice-ordered algebras with a multiplicative identity that is a weak order unit. The lattice-ordered ring $O(\mathcal{Y})$ of all continuous real-valued functions on a topological space $y$ is a $\Phi$-algebra, and it is shown in [5] that every $\Phi$-algebra $A$ is isomorphic to a ring of continuous functions from a compact space $\mathcal{X}$ into the two-point compactification of the real line $R$ such that every $f \in A$ is real-valued on an (open) dense subset of $X$.

If $A=C(\vartheta)$, and $M$ is a maximal $l$-ideal of $A$, it is known that $A / M$ is a real-closed field that is either the real field, or an $\eta_{1}$-set in its unique ordering. We show that for any uniformly closed $\Phi$-algebra $A$, the residueclass fields are real-closed. This result seems to be new even for $\Phi$-algebras of real-valued functions. Stronger assumptions must be made to guarantee that if $A / M$ is not the real field, then it is an $\eta_{1}$-set. We show that if $A$ is closed under countable composition (i.e. if $\left\{f_{n}\right\}$ is a sequence of elements of $A$, and $g \in C\left(R^{\infty}\right)$, then there is an $h \in A$ such that $h(x)$ $=g\left(f_{1}(x), \ldots, f_{n}(x), \ldots\right)$ whenever all of the $f_{n}$ are real-valued $)$, then $\boldsymbol{A}$ is closed under uniform convergence, and $A / M$ is an $\eta_{1}$-set if it is not the real field. In fact, under this hypothesis, $A$ is a homomorphic image of $C(y)$, for some topological space $Y$.

It is shown also that every $\Phi$-algebra $A$ is a homomorphic image of a $\Phi$-algebra $B$ of real-valued functions; moreover, $B$ can be chosen so that it is closed under countable composition, (finite) composition, uniform convergence, or bounded inversion, provided that $A$ is.

An example is given of a uniformly closed $\Phi$-algebra $A$ that is closed. under (finite) composition, with a maximal $l$-ideal $M$ such that $A / M$ contains $R$ properly, and has a countable cofinal subset. This serves to correct an error in [6].

The notation and terminology is that of [5]. An effort has been made to keep the exposition reasonably self-contained.

* This research was supported in part by the Office of Naval Research (U. S. A.). 
1. Residue class fields of uniformly closed $\Phi$-algebras. Recall from [5] that a $\Phi$-algebra $A$ is said to be closed under bounded inversion provided $1 / a \in A$ whenever $a \geqslant 1$ in $A$.

1.1. LEMca. $A$ Ф-algebra $A$ is closed under bounded inversion if and only if every maximal ideal of $A$ is an l-ideal.

Proof. If $a \geqslant 1$ in $A$, then $a$ is in no proper $l$-ideal of $A$. Hence, if every maximal ideal of $A$ is an $l$-ideal, then $A$ is closed under bounded inversion.

For the converse, let $M$ be a maximal ring ideal of $A$ and suppose $b \notin M,|a| \geqslant|b|$. Since $A / M$ is a field, there is an $x \in A$ such that $b x+m=1$. Squaring, we obtain $b^{2} x^{2}+m^{\prime}=1$, where $m^{\prime}=2 b x m+m^{2} \in M$. Thon, since $b^{2} \leqslant a^{2}$, and $x^{2} \geqslant 0$, we must have $a^{2} x^{2}+m^{\prime} \geqslant 1$. If $A$ is closed under bounded inversion, there is a $z \in A$ such that $\left(a^{2} x^{2}+m^{\prime}\right) z=1$, so that $a\left(a x^{2} z\right) \equiv 1(\bmod M)$. Thus $a \notin M$. Hence $M$ is an $l$-ideal of $A$.

As in [5], we say that $A$ is uniformly closed if every Cauchy sequence of elements of $A$ converges to an element of $A$.

If $X$ is any compact space, let $D(X)$ denote the set of all continuous functions defined on $X$ with values in the two point compactification $\gamma R=[-\infty,+\infty]$ of the real line $R$ that are real-valued on a dense (open) subset of $X$. If lattice operations are defined coordinatewise, then $D(X)$ forms a lattice. Let $f, g \in D(X)$. If there is an $h \in D(X)$ such that $h(x)$ $=f(x)+g(x)$ whenever $f(x)$ and $g(x)$ are real, wo write $h=f+g$, and similarly for multiplication. In general, neither $f+g$, nor $f \cdot g$ is define It is true, however, that every $\Phi$-algebra $A$ can be isomorphically repr sented in $D(M(A))$, where $\mathscr{M}(A)$ is the space of maximal $l$-ideals of with the Stone (= hull-kernel) topology. $\mathscr{C}(A)$ is always a compa Hausdorff space ([5], Theorem 2.3). We will regard $A$ as represented this way whenever it is convenient to do so.

We will also utilize the following, proved in [5], 3.2 and 3.7.

1.2. The following properties of $a \Phi$-algebra $A$ are equivalent.

(i) $A$ is uniformly closed.

(ii) $A^{*}$ and $C(M(A))$ are isomorphic.

(iii) $A$ is an order-convex subset of $D(M(A))$.

From (ii), it is evident that every uniformly closed $\Phi$-algebra is closed under bounded inversion.

If $a \in A$, let $\mathscr{R}(a)=\{x \in \mathscr{M}(A):|a(x)|<\infty\}$, let $\mathscr{E}(a)=\{x \in \mathscr{M}(A): a(x)$

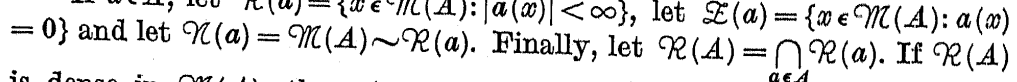
is dense in $M(A)$, then $A$ is called an algebra of real-valued functions.

Let $A$ be any $\Phi$-algebra, and let $g \in C\left(R^{n}\right)$. If, for every $f_{1}, \ldots, f_{n} \in A$, there is an $h \in A$ such that $h(x)=g\left(f_{1}(x), \ldots, f_{n}(x)\right)$, whenever $x \in \bigcap_{i=1}^{n} R\left(f_{i}\right)$, we say that $A$ is closed under composition with $g$, or that $A$ admits $g$. Evidently $h$ is unique; we shall write $h=g\left(f_{1}, \ldots, f_{n}\right)$. Every $\Phi$-algebra admits the constant functions and the projection functions $p_{i}$, where $p_{i}\left(\lambda_{1}, \ldots, \lambda_{n}\right)$ $=\lambda_{i}(i=1,2, \ldots, n)$.

We let $F(A, n)$ denote the family of all $g \in C\left(R^{n}\right)$ that $A$ admits. It is easily verified that $F(A, n)$ is a $\Phi$-algebra if operations are defined in the usual coordinatewise fashion.

If $A$ is uniformly closed, so is $F(A, n)$. For if $\left\{g_{i}\right\}$ is a Cauchy sequence in $F(A, n)$, then it converges to some $g \in O\left(R^{n}\right)$. If $f_{1}, \ldots, f_{n} \in A$, then $\left\{g_{i}\left(f_{1}, \ldots, f_{n}\right)\right\}$ is a Cauchy sequence of elements of $A$ whose limit must be $g\left(f_{1}, \ldots, f_{n}\right)$.

Let $A$ be uniformly closed and let $p=\left(\sum_{i=1}^{n} p_{i}^{2}\right)^{1 / 2}$. Note that $R^{n} \subset M(F(A, n))$, and that $R(p)=R^{n}$. Hence by [5], Lemma 3.5, every $g \in C^{*}\left(R^{n}\right)$ has a continuous extension over $m(F(A, n))$, so $\(F(A, n))$ and $\beta R^{n}$ are homeomorphic. By 1.2 (iii), $F(A, n)$ is an order-convex sub- $\Phi$-algebra of $C\left(R^{n}\right)$. Thus, we have established

1.3. LEMMA. If $A$ is a uniformly closed $\Phi$-algebra, then, for $n=1,2, \ldots$, $F(A, n)$ is a uniformly closed sub- $\Phi$-algebra of $C\left(R^{n}\right)$ containing all $g \in \dot{C}\left(R^{n}\right)$ such that $|g| \leqslant \lambda\left(1+p^{2}\right)^{m}$ for some $\lambda \in R^{+}$, and some positive integer $m$.

Recall that a totally ordered field $F$ is called real-closed if every $a \in F^{+}$has a square root and every polynomial of odd degree with coefficients in $F$ has a zero in $F$.

1.4. THEOREM. If $A$ is a uniformly closed $\Phi$-algebra, and $M \in M(A)$, then $A / M$ is a real-closed field.

Proof. Since $A$ is closed under bounded inversion, Lemma 1.1 shows that $A / M$ is a field. By [5] Theorem 3.8, every $a \in A^{+}$has a square root, so we need only show that polynomials of odd degree with coefficients in $A / M$ have zeros.

Let $p_{\lambda}(w)=w^{m+1}+\lambda_{m} w^{m}+\ldots+\lambda_{0}$ denote a monic polynomial with real coefficients of positive degree. Let $r_{1}(\lambda), r_{2}(\lambda), \ldots, r_{m+1}(\lambda)$ denote the real parts of the complex zeros of $p_{\lambda}(w)$ indexed so that $r_{1}(\lambda) \leqslant r_{2}(\lambda) \leqslant \ldots$ $\leqslant r_{m+1}(\lambda)$. This serves to define $m+1$ real-valued functions on $R^{m+1}$. It is known that each of these functions is continuous ([4]). Moreover, by [9], p. 96, $\left|r_{i}(\lambda)\right|<1+\left|\lambda_{0}\right| \vee\left|\lambda_{1}\right| \vee \ldots \vee\left|\lambda_{m}\right|$ for each $\lambda=\left(\lambda_{0}, \ldots, \lambda_{m}\right) \in R^{m+1}$, and $i=1, \ldots, m+1$. Hence, by Lemma $1.3, A$ is closed under composition with $r_{i}$.

Let $q(w)=w^{2 n+1}+f_{2 n} w^{2 n}+\ldots+f_{0}$ denote a monic polynomial of odd degree with coefficients in $A$. By the above, $s_{i}=r_{i}\left(f_{0} ; \ldots, f_{2 n}\right) \in A$. Since $q(w)$ has odd degree, for each $x \in \bigcap_{i=1}^{2 n+1} R\left(f_{i}\right)$, there is an $i$ such that $q\left(s_{i}\right)(x)=0$. 
Hence $q\left(s_{1}\right) q\left(s_{2}\right) \ldots q\left(s_{2 n+1}\right)=0$. Since $M$ is a prime ideal, there is an $i_{0}$ such that $q\left(s_{i_{0}}\right) \in M$. Hence $A / M$ is a real-closed field.

The argument just given enables us to reach the following slightly stronger conclusion. If $A$ is uniformly closed $\Phi$-algebra, and $P$ is a prime $l$-ideal of $A$, then every positive element of $A / P$ has a square root, and every monic polynomial of odd degree with coefficients in $A / P$ has a zero in $A / P$. Also, as we will show next, the assumption that $P$ is an $l$-ideal is rodundant.

1.5. Lemca. Every prime ideal $P$ of a uniformly closed $\Phi$-algebra $A$ is an l-ideal.

Proof. Since $|c|^{2}=c^{2}$, we know that $o \in P$ if and only if $|c| \in P$. Thus, since $|c|=(|c| \wedge 1)(|c| \vee 1)$, and since, by Lemma 1.2, $A$ is closed under bounded inversion, $c \in P$ if and only if $|c| \wedge 1 \in P$.

Suppose now that $|b| \leqslant|a|$, and $a \in P$. Then $|b| \wedge 1 \leqslant|a| \wedge 1 \in P \cap A^{*}$. But, by Lemma 1.2, $A^{*}$ and $C(M(A))$ are isomorphic, and by [3], Chapt. 14 , every prime ideal of the latter is an $l$-ideal. So $|b| \wedge 1 \in P$, whence $b \in P$. Hence $P$ is an $l$-ideal.

1.6. REMARK. It is remarked in [3], Chapt. 13, that any totally ordered field containing $R$ properly in which exponentiation of positive elements to real powers can be defined has degree of transcendency at least $c$ over $R$. It follows that if $A$ is a uniformly closed. $\Phi$-algebra, and $M \in M(A)$ is hyper-real, then $A / M$ has degree of transcendency at least $c$ over $R$.

If $S$ and $T$ are subsets of a totally ordered sot $L$, and $s<t$ whenever $s \in S$ and $t \in T$, we will write $S<T$.

1.7. THEOREM. Let $P$ be a prime ideal of a uniformly closed $\Phi$-algebra $A$. If $S$ and $T$ are countably infinite subsets of $A / P$ such that $S$ has no largest element, $T$ has no smallest element, and $S<T$, then there is an a $\in A / P$ such that $S<a<T$.

Proof. Since, by 1.5, $P$ is a prime $l$-ideal, $A / P$ is totally ordered, and by 1.2 ff. we may assume that $0 \leqslant S<T \leqslant 1$. By Lemma 1.2 $A^{*} \cong C(M(A))$. Kohls has shown that the conclusion follows in case $A \cong C(\mathcal{Y})$ for any space $Y$ ([8], Theorem 2.6). Since

$$
\frac{A^{*}}{P \cap A^{*}} \cong \frac{A^{*}+P}{P} \subset A / P
$$

the conclusion holds in this case as well.

A totally ordered set $L$ is called an $\eta_{1}-$ set if whenever $S$ and $T$ aro countable subsets of $L$ such that $S<T$, then there is an $a \in L$ such that $s<a<T$. In particular, an $\eta_{1}$-set has no countable cofinal or coinitial subset.

For any topological space $\mathcal{H}$, and any hyper-real maximal ideal $M$ of $C(Y)$, it is known that $O(Y) / M$ is an $\eta_{1}$-set. Example 1.9 below shows strongly that no comparable conclusion holds for arbitrary uniformly closed $\Phi$-algebras.

Most of the remainder of the paper will be devoted to a discussion of the extra hypotheses needed to conclude that $A / M$ is an $\eta_{1}$-set.

A $\Phi$-algebra $A$ is said to be closed under (finite) composition if $F(A, n)$ $=O\left(R^{n}\right)$ for $n=1,2, \ldots$; that is, if $A$ admits every $g \in C\left(R^{n}\right)$.

As in [5], $A$ is said to be closed under l-inversion if $\langle a\rangle=A$ whenever $\mathscr{I}(a) \subset \chi(b)$ for some $b \in A$. (Recall that $\langle a\rangle$ is the smallest $l$-ideal of $A$ containing a.)

1.8. LEMMA. Let $A$ be a $\Phi$-algebra.

(i) If $F(A, 2)=C\left(R^{2}\right)$ (in particular, if $A$ is closed under composition), then $A$ is closed under l-inversion.

(ii) If $A$ is closed under uniform convergence and l-inversion, then $A$ is closed under composition.

Proof. (i) Let $a, b \in A$, and suppose that $\mathscr{I}(a) \subset \Re(b)$. Let $h=|a| \vee|b|$ let $B_{h}=\{f \in A: \mathcal{R}(f) \supset \mathscr{R}(h)\}$, and let $\mathcal{H}=\left\{(a(x), b(x)) \in R^{2}: x \in \mathscr{R}(h)\right\}$. If $(0, q) \in \mathscr{H}^{-}$, then there is a sequence $\left\{x_{n}\right\}$ of points of $\mathcal{R}(h)$ such that $a\left(x_{n}\right) \rightarrow 0$ and $b\left(x_{n}\right) \rightarrow q$. Since $\Pi(A)$ is compact, $\left\{x_{n}\right\}$ has a limit point $x \in \mathcal{M}(A)$. Clearly $a(x)=0$, and $b(x)=q$, contrary to the assumption that $\mathscr{I}(a) \subset \Re(b)$. Thus, the function $g$ defined on $\Re^{-}$by letting $g(p, q)=1 / p$ is continuous. By the Tietze extension theorem, it has an extension $\bar{g} \in C\left(R^{2}\right)$. Since $F(A, 2)=C\left(R^{2}\right)$, this shows that $1 / a \in A$.

(ii) Suppose that $f_{1}, \ldots, f_{n} \in A$, let $h=\left|f_{1}\right| \vee \ldots \vee\left|f_{n}\right|$, and let $B_{h}=\{a \in A: \mathcal{R}(a) \supset \mathcal{R}(h)\}$. By [5], Theorem 5.8, since $A$ is closed under uniform convergence and $l$-inversion, $B_{h}$ and $C(\mathcal{R}(h))$ are isomorphic. Hence, for any $g \in O\left(R^{n}\right), g\left(f_{1}, \ldots, f_{n}\right) \in A(n=1,2, \ldots)$. Thus, $A$ is closed under composition.

In [6], Theorem 1.28, Isbell states that if $A$ is an algebra of realvalued functions closed under uniform convergence and composition, and $M \in \Pi(A)$ is hyper-real, then $A / M$ is an $\eta_{1}$-set. While he establishes correctly the conclusion of Theorem 1.7 above, $A / M$ may have a countable cofinal subset, as is shown by the following. For $a \in A$, the image of $a$ in $A / M$ is denoted by $M(a)$.

1.9. EXAMPLE. There exists a uniformly closed $\Phi$-algebra $A$, closed under composition, and a hyper-real $M \in M(A)$ such that $A / M$ has a countable cofinal subset.

Proof. Let 9 denote the space of irrational numbers in $(0,1)$ with its usual topology. Since $\beta \mathcal{G}$ is the largest compactification of $\vartheta$, there is a continuous mapping $\pi$ of $\beta \mathcal{G}$ onto $[0,1]$ keeping $\subseteq$ pointwise fixed. Let $\mathscr{Z}_{0}=\pi^{-1}(0)$, and for $i=1,2, \ldots$, let $\delta_{i}=\left\{1 / p^{i} \epsilon(0,1): p\right.$ a prime; $j$ a positive integer, $j \leqslant i\}$, let $\mathscr{L}_{i}=\mathscr{I}_{0} \cup \pi^{-1}\left(\delta_{i}\right)$, and let $\mathscr{U}_{i}=\beta \mathcal{G} \sim \mathscr{E}_{i}$. 
Observe that $\mathscr{S \subset} \mathcal{Y}_{i+1} \subset \mathcal{Y}_{i}$ for $i=1,2, \ldots$, and let $A_{i}=\{f \in D(\beta ९)$ : $\left.\mathcal{R}(f) \supset \mathscr{Y}_{i}\right\}$. Since $\mathscr{Y}_{i}$ contains $\mathscr{O}$, it is $C^{*}$-imbedded in $\beta \subseteq$, so $A_{i}$ and $C\left(\mathscr{Y}_{i}\right)$ are isomorphic. Finally, let $A=\bigcup_{i=1}^{\infty} A_{i}$.

If $\left\{f_{n}\right\}$ is a Cauchy sequence of elements of $A$, then (as is noted in [5], 3.1) $\mathcal{R}\left(f_{n}\right)=\mathscr{R}\left(f_{n+1}\right)=\ldots$ for all but finitely many of the $f_{n}$. Thus we may assume that $\left\{f_{n}\right\} \subset A_{i}$ for some $i$, whence $\left\{f_{n}\right\}$ converges. Similarly, any finite number of elements of $A$ is contained in some $A_{i}$. Thus $A$ is closed under uniform convergence and $l$-inversion.

If every point of the compact space $\mathfrak{Z}_{0}$ had a neighborhood moeting only finitely many of the sets $\left\{\mathscr{E}_{i+1} \sim \mathfrak{E}_{i}\right\}$, then $\mathfrak{E}_{0}$ itsolf would have such a neighborhood. But every neighborhood of 0 meets infinitely many of the sets $\delta_{i+1} \sim \delta_{i}$, so this cannot be the case. Hence, there is an $x \in \mathfrak{E}_{0}$ such that every neighborhood of $x$ meets infinitely many of the sets $\left\{\mathscr{I}_{i+1} \sim \mathscr{L}_{i}\right\}$. By a suitable change of notation, we may assume that every neighborhood of $x$ meets all such sets.

Now each $\mathfrak{Z}_{i}$ is the inverse image of a closed subset of a metrizable space, and hence is a closed $G_{\delta}$. Hence there is an $f_{i} \in A_{i}^{+}$such that $\eta\left(f_{i}\right)=\mathscr{L}_{i}$. Now, $M_{x}\left(f_{1}\right)$ is greater than all the constant functions, so $M_{0}$ is hyper-real. If $g \in A$, there is an $i$ such that $\chi(g) \subset \mathscr{E}_{i}$. Suppose there were an $h \in M_{x}$ such that $g+h \geqslant f_{i+1}$. Then $\chi\left(f_{i+1}\right) \subset \chi(g) \cup \chi(h)$, and hence $\chi(h) \supset \chi\left(f_{i+1}\right) \sim \chi(g) \supset \mathscr{I}_{i+1} \sim \mathscr{E}_{i}$. But this latter set has $x$ as a limit point, contrary to the fact that $h \in M_{x}$. We conclude that $\left\{M_{x}\left(f_{i}\right): i=1,2, \ldots\right\}$ is a countable cofinal subset of $A / M_{x}$.

2. $\Phi$-algebras closed under countable composition. The example of the last section motivates the consideration of a more re stricted class of $\Phi$-algebras.

We designate a countable product of copies of $R$ as $R^{\infty}$.

Let $A$ be a $\Phi$-algebra, and suppose that for every $g \in \dot{O}\left(R^{\infty}\right)$, and every sequence $\left\{f_{n}: n=1,2, \ldots\right\}$ of elements of $A$, there is an $h \in A$ such that $h(x)=g\left(f_{1}(x), \ldots, f_{n}(x), \ldots\right)$ whenever $x \in \bigcap_{n=1}^{\infty} R\left(f_{n}\right)$; we say that $A$ is closed under countable composition. By the Baire category theorem, $\bigcap_{n=1}^{\infty} R\left(f_{n}\right)$ is dense in $M(A)$, so $h$ is unique. We denote it by $g\left(f_{1}, f_{2}, \ldots, f_{n}, \ldots\right)$.

Clearly, if $A$ is closed under countable composition, it is closed under composition, and hence, by Lemma 1.8 , it is closed under $l$-inversion. This motivates the consideration of the following concept.

A $\Phi$-algebra $A$ is said to be closed under countable l-inversion provided that $\langle g\rangle=A$ for each $g \in A$ for which there is a sequence $\left\{f_{n}: n=1,2, \ldots\right\}$ of elements of $A$ such that $\mathscr{X}(g) \subset \bigcup_{n=1}^{\infty} \Upsilon\left(f_{n}\right)$.
The relationship between these two latter concepts is given by

2.1. THEOREM. $A$ $\Phi$-algebra $A$ is closed under countable composition if and only if it is uniformly closed and closed under countable l-inversion.

Proof of necessity. Suppose that $A$ is closed under countable composition, and that $Z(g) \subset \bigcup_{n=1}^{\infty} \eta\left(f_{n}\right)$ for some $g, f_{1}, \ldots, f_{n}, \ldots$ in $A$. Let $g=f_{0}, \quad \mathcal{f}=\bigcap_{n=0}^{\infty} \mathcal{R}\left(f_{n}\right)$, and define $\psi: \mathcal{H} \rightarrow R^{\infty}$ by letting $\psi(y)$ $=\left(f_{0}(y), f_{1}(y), \ldots, f_{n}(y), \ldots\right)$ for all $y \in \mathcal{Y}$. Let $\mathcal{X}$ denote the closure in $R^{\infty}$ of $\psi[\mathcal{Y}]$. If $x=\left(x_{0}, x_{1}, \ldots, x_{n}, \ldots\right) \in \mathcal{H}$, then $x_{0} \neq 0$. For, otherwise there would be a sequence $\left\{y_{n}\right\}$ of points of $\gamma$ such that $\psi\left(y_{n}\right)$ converges to $x$. Since $\Pi(A)$ is compact, $\left\{y_{n}\right\}$ has an accumulation point in $\Pi(A)$, which is a point of $Z(g)$ not in $\bigcup_{n=1}^{\infty} \chi\left(f_{n}\right)$.

Hence the function $r: \mathcal{l} \rightarrow R$ defined by letting $r\left(x_{0}, x_{1}, \ldots, x_{n}, \ldots\right)$ $=1 / x_{0}$ is well-defined and continuous. By the Tietze extension theorem ([7], p. 242), $r$ has an extension $s \in C\left(R^{\infty}\right)$. Since $A$ is closed under countable composition, $s\left(f_{0}, f_{1}, \ldots, f_{n}, \ldots\right)$ is an element $h$ of $A$ such that $g h=1$ on the dense subset $\mathcal{Y}$ of $\mathscr{K}(A)$. Thus $h$ is the inverse of $g$, whence $\langle g\rangle=A$.

Suppose next that $\left\{f_{n}\right\}$ is a Cauchy sequence of elements of $A$, define $\mathcal{Y}$ as above, define $\psi$ : $\mathcal{Y} f \rightarrow R^{\infty}$ by letting $\psi(y)=\left(f_{1}(y), \ldots, f_{n}(y), \ldots\right)$ for all $y \in \mathcal{Y}$, and let $\mathcal{Y}$ denote the closure of $\psi[\mathcal{Y}]$ in $R^{\infty}$.

Since $\left\{f_{n}\right\}$ is a Cauchy sequence, for every $\varepsilon>0$ there is a positive integer $m$ such that for every $x=\left(x_{1}, x_{2}, \ldots, x_{n}, \ldots\right)$ of $\psi[\mathcal{Y}],\left|x_{p}-x_{q}\right|<\varepsilon$ whenever $p, q \geqslant m$. For any $z \in \mathcal{H}$, if $p, q \geqslant m$, then $\left|z_{p}-z_{q}\right| \leqslant \varepsilon$. For, if not, for some such $z, p$, and $q$, there is a $\delta>0$ such that $\left|z_{p}-z_{q}\right|=\varepsilon+2 \delta$. Then $\left\{w \in R^{\infty}:\left|w_{p}-z_{p}\right|<\delta\right.$ and $\left.\left|w_{q}-z_{q}\right|<\delta\right\}$ is a neighborhood of $z$ in $R^{\infty}$ that contains no point of $\psi[\mathcal{Y}]$, contrary to the fact that $z \in \mathcal{H}$. Hence, for each $z \in \mathcal{H},\left\{z_{n}\right\}$ is a Cauchy sequence. Define $s: \mathcal{H} \rightarrow R$ by letting $s(z)=\lim _{n \rightarrow \infty} z_{n}$. It is easily verified that $s \in C(\mathcal{H})$. By the Tietze extension

theorem, $s$ has a continuous extension $t \in C\left(R^{\infty}\right)$. Since $A$ is closed under countable composition, $h=t\left(f_{1}, f_{2}, \ldots, f_{n}, \ldots\right) \in A$. Clearly $\left\{f_{n}\right\}$ converges to $h$. This completes the proof of the necessity.

Before proving the sufficiency, we prove two lemmas that are of independent interest.

Recall that a topological space of is called a Lindelöf space if every open cover of $y$ has a countable subcover,

2.2. LEMra. Let $\mathcal{Y}$ be a subspace of a compact space $X$ such that for some countable family $\Psi$ of closed subsets of $X$, for every pair of points $p \in \mathcal{Y}, q \in \mathcal{X} \sim \mathcal{Y}$ there is a set in $\Psi$ containing $p$ but not $q$. Then $\mathcal{Y}$ is a lindelöf space. 
Proof. Let $\left\{\mathscr{U}_{a}: \alpha \in T\right\}$ denote an open cover of $y$. For oach $\alpha \in I$,

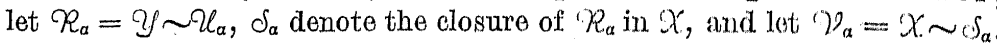
Clearly $\mathcal{V}_{\alpha} \cap y=\mathcal{U}_{\alpha}$, and the sets $\eta_{\alpha}$ cover $X \sim \eta$, where $\chi=\cap\left\{\delta_{\alpha}\right.$ : $\alpha \in \Gamma$ \}. Clearly $\mathcal{H}$ is a compact subset of $X \sim Y$.

Let $\mathcal{F}$ denote the union of all those subsets of $\mathcal{X}$ that are disjoint from $\mathcal{H}$, and are finite intersections of elements of $\Psi$. Then $\%$ is $\sigma$-compact, and hence is a Lindelöf space. Thus, it suffices to show that y C T. But, for each $p \in$ If, by hypothesis, the intersection of all the olemonts of $\psi$ containing $p$ is disjoint from $x \subset X \sim \mathcal{C}$. Fence some finite intersection of them is disjoint from $\mathcal{x}$. Hence $y \subset \mathcal{F}$.

2.3. Corollary. Every subset of a compact space $X$ that is in the smallest family of subsets of $X$ containing the closed subsets and olosed under countable union and intersection, is a Lindelöf space. In particular, for any $\Phi$-algebra $A$ and any sequence $\left\{f_{n}\right\}$ of elements of $A, \bigcap_{n=1}^{\infty} R\left(f_{n}\right)$ is a tindelöf space.

Proof. Every closed subspace of $X$ satisfies the hypothesis of Lemma 2.2, so it sufficies to show that if $\varepsilon_{n}$ satisfies this latter condition with associated countable family of closed sets $\Psi_{n}$ for $n=1,2, \ldots$, then so does $\mathcal{Y}=\bigcup_{n=1}^{\infty} \varepsilon_{n}$, and $\mathfrak{Z}=\bigcap_{n=1}^{\infty} \varepsilon_{n}$. If $p \in \mathscr{Y}, q \in X \sim \mathcal{Y}$, then $p \in \mathcal{E}_{n}$ for some $n$, and $q \notin \mathcal{E}_{n}$, so there is an element of $\Psi_{n}$ that contains $p$ and not $q$. Thus, $Y$ satisfies the hypothesis of Lemma 2.2 with associated countable family of closed sets $\bigcup_{n=1}^{\infty} \Psi_{n}$. The proof for $\mathscr{Z}$ is similar.

2.4. LEMMA. Let $A$ be a uniformly closed $\Phi$-algebra that is closed under countable l-inversion, let $\left\{f_{n}\right\}$ be a sequence of elements of $A$, let $Y=\bigcap_{n}^{\infty} \mathcal{R}\left(f_{n}\right)$, and let $B=\{g \in A: \mathcal{Y} \subset \mathcal{R}(g)\}$. Then $B$ and $O(\mathcal{Y})$ are isomorphic.

Proof. Clearly $B$ is a sub- $\Phi$-algebra of $A$. Since $B \supset A^{*}, \eta(B)$ $=\prod(A)$, and since $A$ is uniformly closed, so is $B$. Since $f_{n} \in B$ for $n=1,2, \ldots, \mathcal{R}(B)=y . B$ is also closed under inversion of elements without zeros in $\Re(B)$. For, if $Z(g) \cap \chi(B)=\varnothing$, then $\mathscr{Z}(g) \subset \bigcup_{n=1}^{\infty} \Re\left(f_{n}\right)$, so, since $A$ is closed under countable l-inversion, $1 / g$ is in $A$ and is real-valued on $\mathcal{Y}$. By Corollary 2.3, $y$ is a Lindelöf space, so by [5] Lemma 5.3, for every $h \in O(\mathcal{O})$, there is a $b \in C^{*}(\eta /(B))$ such that $h^{-1}(0)$ $=\mathscr{E}(b) \cap \mathcal{Y}$. It follows from [5], Theorem 5.2 that $B$ and $O(R(B))$
are isomorphic.

The proof of sufficiency for Theorem 2.1 is now easy in view of Lemma 2.4. If $\left\{f_{n}\right\}$ is any sequence of elements of a uniformly closed $\Phi$-algebra that is closed under countable $l$-inversion, then, by Lemma 2.4 , if $y=\bigcap_{n=1}^{\infty} \mathcal{R}\left(f_{n}\right)$, then $C(\mathcal{Y})$ is a subalgebra of $A$. So, for any $g \in C\left(R^{\infty}\right)$, $g\left(f_{1}, \ldots, f_{n}, \ldots\right)$ is in $A$.

Before returning to hyper-real residue class fields, we prove

2.5. THEOREM. Every $\Phi$-algebra $A$ can be obtained as a homomorphio image of $a \Phi$-algebra $B$ of real-valued functions in such a way that if $A$ is uniformly closed, or closed under bounded inversion, or composition, or countable composition, then so is $B$.

Proof. $B$ will be defined as an algebra of continuous real-valued functions on $\dddot{M}(A) \times \chi$ where $\chi$ is the discrete space of positive integers. Every element $g$ of $B$ will be regarded as a sequence $\left\{g_{n}\right\}$ of functions on $\mathscr{M}(A)$, where $g_{n}(p)=g(p, n)$, for all $p \in \mathscr{M}(A)$. $B$ consists precisely of all those $\left\{g_{n}\right\}$ which converge pointwise to an element of $A$ on a dense $G_{\delta}$; i.e. those $g \in C(\Re(A) \times \eta)$ such that for some $f \in A$, and for some dense $G_{\delta}$-set $\mathscr{Y} \subset \mathcal{C}(A)$, for each $p \in \mathcal{Y}$, the sequence $\{g(p, n)\}$ of real numbers converges in $\gamma R=[-\infty,+\infty]$ to $f(p)$.

Since the intersection of two dense $G_{\delta}$-sets is dense, each $g \in B$ converges to a unique $\lambda(g) \in A$. Similarly, it is easily verified that $B$ is a $\Phi$-algebra, and that $\lambda$ is a homomorphism of $B$ into $A$. Moreover, if $f \epsilon A$, and $g_{n}=(f \wedge n) \vee(-n)$ for $n=1,2, \ldots$, then $g_{n}(p)$ converges to $f(p)$ for all $p \in \eta(A)$. Hence $\lambda(g)=f$, so $\lambda$ is a homomorphism of $B$ onto $A$.

Suppose that $A$ is closed under countable composition, that $\left\{g_{n}\right\}$ is a sequence of elements of $B$, and that $h \in C\left(R^{\infty}\right)$. For $n=1,2, \ldots$, there is a dense $G_{\delta}$-set $\mathcal{Y}_{n}$ in $M(A)$ such that for each $p \epsilon \rho_{n}, g_{n}(p, m)$ converges to $\lambda\left(g_{n}\right)(p)$. Let $\mathcal{Y}$ denote the intersection of all the $\gamma_{n}$ and all $\chi\left(\lambda\left(g_{n}\right)\right)$, for $n=1,2, \ldots$ Since $\Pi(A)$ is compact, this countable intersection of dense $G_{\delta}$-sets is a dense $G_{\delta}$-set. Moreover, each $\lambda\left(g_{n}\right)$ is real-valued on $\mathcal{Y}$, and so is $h\left(\lambda\left(g_{1}\right), \ldots, \lambda\left(g_{n}\right), \ldots\right) \in A$. For each $p \in \mathcal{Y}$, and for each $n$, the real numbers $g_{n}(p, m)$ converge to $\lambda\left(g_{n}\right)(p)$. Then the points $\left\{x_{m}\right\}$ of $R^{\infty}$, whose $n$-th coordinates are $g_{n}(p, m)$, form a convergent sequence in $R^{\infty}$, whose limit $z$ has as $n$-th coordinate $\lambda\left(g_{n}\right)(p)$. Since $h$ is continuous, $h\left(x_{m}\right) \rightarrow h(z)$. Thus, the well-defined continuous function $h\left(g_{1}, \ldots, g_{n}, \ldots\right)$ in $C(\Re(A) \times \Upsilon)$ is in $B$, since it converges pointwise on $y$ to $h\left(\lambda\left(g_{1}\right), \ldots, \lambda\left(g_{n}\right), \ldots\right)$. That is, $B$ is closed under countable composition.

Simplified versions of the preceding establish the remaining assertions.

In [1], 2.1, Corson and Isbell show that if an algebra $A$ of real-valued functions is closed under countable composition, then it is closed under composition for all higher cardinals. This fact may be used to establish the following.

Fundamenta Mathematicae, T. L (1961) 
2.6. THEOREM. Every $\Phi$-algebra A closed under countable composition is a homomorphic image of $C(\mathcal{Y})$ for some topological space $Y$.

For, by Theorem 2.5, we may assume without loss of generality that $A$ is an algebra of real-valued functions. Let of denote the cartesian product of as many copies $R_{f}$ of $R$ as there are elements $f$ of $A$. Let $e$ denote the mapping of $R(A)$ into $\mathcal{Y}$ such that the $f$-th coordinate $e(x)_{f}$ of $e(x)$ is $f(x)$. Finally, let $\tau g=g \cdot e$ for each $g \in O(Y)$. By the result cited above, since $A$ is closed under countable composition, and hence unlimited composition, $\tau g \in A$ for all $g \in O(Y)$. Clearly $\tau$ is a homomorphism of $O(\vartheta)$ onto $A$.

In [2], it is shown that if $M$ is a hyper-real maximal ideal of $O(Y)$, for some topological space $Y$, then $O(Y) / M$ is an $\eta_{1}$-sot. Honce, by Theorems 1.4 and 2.6, we have immediately

2.7. CoRollarr. If $A$ is a $\Phi$-algebra closed under countable composition, and $M \in \mathcal{O}(A)$ is hyper-real, then $A / M$ is real-closed field that is an $\eta_{1}-$ set.

2.8. Corollary. If $A=D(\Pi(A))$ is a $\Phi$-algebra, and $M \in M(A)$ is hyper-real, then $A / M$ is a real-closed field that is an $\eta_{1}$-set.

Proof. By 2.1 and 2.7, it suffices to show that the $\Phi$-algebra $A=D(M(A))$ is closed under countable $l$-inversion and uniform convergence. The latter follows immediately from Lemma 1.2. Lot $\left\{f_{n}\right\}$ be

a sequence of elements of $A$ such that $Z(g) \subset \bigcup_{n=1}^{\infty} \eta\left(f_{n}\right)$. Then $\mathscr{E}(g)$ is nowhere dense and $g$ cannot be a divisor of zero. Thus, by [5], Theorem 3.9 , $1 / g \in A$.

In [2], it is shown that all real-closed $\eta_{\alpha}$-fields of power $\kappa_{\alpha}$ are isomorphic, if $\alpha>0$. It follows from Corollary 2.8 that, if $\kappa_{1}=0$, then all of the residue class fields of the $\Phi$-algebra of all Lebesgue measurable functions on $R$, modulo the ideal of functions vanishing off sets of measure zero, are isomorphic. See [5], Corollary 3.10.

\section{References}

[1] H. H. Corson and J. R. Isbell, Some properties of strong wniformities, Quart. J. Math. (Oxford) (2) 11 (1960), pp. 17-33.

[2] P. Erdös, L. Gillman, and M. Henriksen, An isomorphism theorem for real-closed fields, Ann. of Math. 61 (1955), pp. 542-554.

[3] L. Gillman and M. Jerison, Rings of continuous funotions, Princeton, N. J. 1960

[4] M. Henriksen and J. R. Isbell, On the continuity of the real roots of an algebraic equation, Proc. Amer. Math. Soc. 4 (1953), pp. 431-434

[5] - and D. G. Johnson, On the structure of a class of arohimedean lattioeordered algebras, Fund. Math., this volume, pp. 73-94
[6] J. R. Isbell, Algebras of uniformly continuous functions, Ann. of Math. 68 (1958), pp. 96-125.

[7] J. L. Kelley, General topology, New York 1955.

[8] C. W. Kohls, Prime ideals in rings of continuous functions. Illinois J. Math. 2 (1958), pp. 505-536

[9] M. Marden, The geometry of the zeros of a polynomial in a complex variable, American Mathematical Society, New York 1949.

PURDUE UNIVERSITY AND

PENNSYLVANIA STATE UNIVERSITY

Règu par la Rédaction le 1. 9. 1960 